\title{
EXTRAÇÕES DE ÓLEOS DE SEMENTES DE CITROS E SUAS ATIVIDADES SOBRE A FORMIGA CORTADEIRA Atta sexdens E SEU FUNGO SIMBIONTE
}

João B. Fernandes*, Valmir David, Patrícia H. Facchini, M. Fátima das G. F. da Silva, Edson Rodrigues Filho e Paulo C. Vieira

Departamento de Química, Universidade Federal de São Carlos, CP 676, 13565-905 São Carlos - SP

Mário S. Galhiane

Departamento de Química, Universidade Estadual Paulista, Av. Luiz Edmundo C. Coube, s/n, 17033-360 Bauru - SP. Fernando C. Pagnocca, Odair C. Bueno, M. José Hebling, Sandra R. Victor e Ana Maria Rodrigues dos Santos Centro de Estudos de Insetos Sociais, Universidade Estadual Paulista, Campus de Rio Claro, CP 199, 13506-900 Rio Claro - SP

Recebido em 23/10/01; aceito em 7/3/02

\begin{abstract}
CITRUS SEED OILS EXTRACTIONS AND THEIR ACTIVITY AGAINST LEAF CUTTING ANT Atta sexdens AND ITS SYMBIOTIC FUNGUS. Seeds Citrus oils (C. sinensis, $C$. limon and C. reticulata) extraction with hexane in a soxhlet apparatus and through supercritical fluid $\left(\mathrm{CO}_{2}\right)$ were done. Besides triglycerides, the oils obtained with hexane comprised volatile compounds such as terpenes and fatty alcohols, esters, and aldehydes. However, the oils obtained by extraction with supercritical fluid presented only triglycerides. These results indicate that the extraction using supercritical fluid presents better selectivity. The activity of the oils on the development of the ant symbiotic fungus, Leucoagaricus gongylophorus, showed week activity and the topic insecticide assay showed better activity for the tangerine seed oil.
\end{abstract}

Keywords: supercritical fluid extraction; Citrus oils; ants.

\section{INTRODUÇÃO}

A produção de frutos cítricos no Brasil tem aumentado nos últimos vinte anos, passando de 40 para 260 milhões de caixas, levando o Brasil a ser o líder mundial em exportação de suco de laranja concentrado, com a participação de $71 \%$ do mercado internacional ${ }^{1,2}$.

Sementes secas, sem o tegumento (cerca de $60 \%$ do peso total), podem produzir entre 50 a $55 \%$ de óleo. Esta percentagem pode variar em função da espécie de fruto e da técnica usada para extração. A extração com hexano produz geralmente de 30 a $35 \%$ de óleo, mas pode chegar a $57 \%$. Laranjas do tipo valenciana produzem $0,317 \mathrm{~kg}$ de sementes por caixa (cerca de $40,8 \mathrm{~kg}$ ) e a indústria processa 200.000 caixas por mês, o que equivale a $63.400 \mathrm{~kg}$ de sementes por mês ou $760.800 \mathrm{~kg}$ por $\mathrm{ano}^{3}$. Atualmente, as sementes e as cascas de citros são misturadas com bagaço e são empregados como complemento para alimentação animal. Portanto, a utilização de sub-produtos da indústria de citros é altamente desejável. Os componentes das sementes devem ser melhor estudados a fim de se obter seus constituintes puros ou frações que tenham alto valor agregado.

Tradicionalmente, para se extrair o óleo de sementes vegetais empregam-se extrações com solventes orgânicos ou por prensagem das sementes, sendo que este último processo produz menor quantidade que o primeiro. Extração usando fluido supercrítico é relatada na literatura, sendo o fluido de $\mathrm{CO}_{2}$ o mais utilizado e a extração é dependente da pressão, temperatura e da granulação do pó das sementes ${ }^{4}$.

A associação mutualística entre a formiga cortadeira Atta sexdens (saúva limão) e o fungo Leucoagaricus gongilophorus é muito forte e existe uma total dependência entre ambos. Nesta interação as formigas usam vegetais frescos para o desenvolvimento do fungo e para a própria alimentação ${ }^{6}$ e, de acordo com Fisher e colaboradores ${ }^{7,8} \mathrm{e}$ Quinlan e Cherrett ${ }^{9}$, o fungo constitui a única fonte de alimento para as larvas das formigas, além de fornecer cerca de $9 \%$ da energia

*e-mail: djbf@power.ufscar.br necessária para as operárias das formigas ${ }^{10}$.

As formigas cortadeiras constituem sério problema à agricultura brasileira $^{5}$ e elas têm sido controladas, principalmente, utilizando-se inseticidas de origem sintética, os quais agem indiscriminadamente contra todos os insetos, inclusive os benéficos.

Compostos com maior especificidade (dirigidos ao controle das formigas cortadeiras), por ação fungicida, formicida ou ambos são bastante desejáveis ${ }^{11}$.

Em estudos prévios, realizados pelo grupo de produtos naturais da UFSCar e o Centro de Estudo de Insetos Sociais da UNESP-Rio Claro $^{12,13}$, foi observada a ação tóxica de extratos de plantas, seus ácidos graxos, assim como a associação de ácidos graxos com triglicerídeos sobre as operárias das formigas cortadeiras e sobre o seu fungo simbionte.

O objetivo deste trabalho foi avaliar a extração de óleos das sementes de plantas cítricas com hexano em aparelho "soxhlet" e por fluido supercrítico de $\mathrm{CO}_{2}$, através da utilização de um equipamento comercial e um construído no laboratório ("home made"), assim como avaliar o efeito dos extratos obtidos no desenvolvimento do fungo Leucoagaricus gongylophorus, simbionte de formigas cortadeiras e também quanto à atividade inseticida por aplicação tópica.

\section{PARTE EXPERIMENTAL}

\section{Coleta de sementes}

As sementes de laranja (Citrus sinensis), limão siciliano ( $C$. limon) e tangerina (C. reticulata) foram coletadas na área de descarte do extrator industrial, juntamente com os bagaços dos frutos. As sementes foram separadas dos outros materiais manualmente. Em seguida elas foram lavadas com água e colocadas para secar em uma estufa de circulação à temperatura de $50-55^{\circ} \mathrm{C}$ até peso constante (cerca de 3 dias). As sementes secas foram moídas em um moinho de hélices e peneiradas com uma malha de $2 \mathrm{~mm}$. 


\section{Extração dos óleos com solvente}

Os óleos das sementes foram obtidos através de extrações com hexano $(300 \mathrm{~mL})$ seguido por diclorometano $(300 \mathrm{~mL})$ em um aparelho "soxhlet" com balão de $500 \mathrm{~mL}$ de capacidade e condensador de bola de $25 \mathrm{~cm}$ de altura por $5 \mathrm{~cm}$ de diâmetro interno. O processo foi repetido três vezes para cada solvente e os refluxos foram em intervalos de cerca de $10 \mathrm{~min}$, durante $3 \mathrm{~h}$.

\section{Extração dos óleos com fluido supercrítico}

Extrator "home made"

O extrator contém coluna de aço de 1 polegada de diâmetro com parede de $4,5 \mathrm{~mm}$, altura de $670 \mathrm{~mm}$ e $300 \mathrm{kgf} / \mathrm{cm}^{2}$ de resistência de carga e capacidade para $150 \mathrm{~g}$ de material em pó; 2 bombas de alta pressão Milton Roy modelo 92014903 operando entre 0,77 a 15,3 $\mathrm{cm}^{3} /$ min e pressão máxima de $408 \mathrm{kfg} / \mathrm{cm}^{2}$, banho termostatizado Neslab modelo RTE-211 operando entre -30 a $100{ }^{\circ} \mathrm{C} ; 3$ válvulas, Tescon 26-1700, 755CSST-04 e 562FI4DXED para retenção e controle de pressão; 4 trocadores de calor ( $7 \mathrm{~m}$ de tubo de $1 / 8$ polegada de diâmetro e parede de $1 \mathrm{~mm} ; 4$ manômetros Willy Dresser com tubo Bourbon classe A2-ABNT; 5 "termopars" modelos TIM-20-JS304-30-I40-02-00-C2000-02 com medidor digital de temperatura S200 modelo S205-J6-AAI-N-1; medidor de fluido Omel Instrumentação e Controles Ltda modelo 4A-0802Z e 2 cilindros de gases $\mathrm{CO}_{2}$ e $\mathrm{N}_{2}$ White Martins ( $25 \mathrm{~kg}$ cada). O sistema foi limpo com etanol antes de ser usado nas extrações.

As condições para extrair $145 \mathrm{~g}$ de semente em pó foram: 1, $15^{\circ} \mathrm{C}$ e $85.10^{5} \mathrm{~Pa} ; 2,40{ }^{\circ} \mathrm{C}$ e $125.10^{5} \mathrm{~Pa}$ e $3,60{ }^{\circ} \mathrm{C}$ e $150.10^{5} \mathrm{~Pa}$, durante $8 \mathrm{~h}$.

\section{Extrator comercial}

Um extrator por fluido supercrítico ISCO modelo SFX 220 (Bomba de seringa DX 100, Sistema de controle de dados SFX 200) foi usado operando entre 1 a $700 \mathrm{kgf} / \mathrm{cm}^{2}$ e 0 a $100{ }^{\circ} \mathrm{C}$.

As condições para extrair $4 \mathrm{~g}$ de sementes em pó foram: $4,40{ }^{\circ} \mathrm{C}$, 280 . $10^{5} \mathrm{~Pa}, 60 \mathrm{~min} ; 5,40{ }^{\circ} \mathrm{C}, 420.10^{5} \mathrm{~Pa}, 12 \min$ e $6,40{ }^{\circ} \mathrm{C}, 490$. $10^{5} \mathrm{~Pa}, 8 \mathrm{~min}$.

\section{Cromatografia gasosa e espectrometria de massas}

As análises de CG/FID e CG/EM foram realizadas em um sistema de cromatografia Marca Varian - Modelo 3400 conectado ao espectrômetro de massas modelo Saturn I, usando a técnica de impacto eletrônico (IE) em $70 \mathrm{eV}$. A coluna capilar utilizada no CG foi de $100 \%$ dimetilpolisosiloxano com ligações entrecruzadas; sílica fundida, $30 \mathrm{~m}$ x 0,25 mm x 0,8 $\mu \mathrm{m}$ de filme). Hidrogênio foi usado como gás de arraste com fluxo de $1,0 \mathrm{~mL} \mathrm{~min}^{-1}$. Condições: $\mathbf{1}$, programa de temperatura no forno: $5 \mathrm{~min}$ a $40{ }^{\circ} \mathrm{C}$, de $40{ }^{\circ} \mathrm{C}$ a $240{ }^{\circ} \mathrm{C}$ a $3{ }^{\circ} \mathrm{C} \mathrm{min}{ }^{-1}$ seguida por um período isotérmico de $5 \mathrm{~min} ; 2$, programa de temperatura no forno: $5 \mathrm{~min}$ a $40{ }^{\circ} \mathrm{C}$, de $40{ }^{\circ} \mathrm{C}$ a $90{ }^{\circ} \mathrm{C}$ a $3{ }^{\circ} \mathrm{C} \cdot \mathrm{min}^{-1}$, $90{ }^{\circ} \mathrm{C}$ a $290{ }^{\circ} \mathrm{C}$ a $8{ }^{\circ} \mathrm{C} \mathrm{min}^{-1}$ seguido por um período isotérmico de $5 \mathrm{~min}$; 3, programa de temperatura no forno: $5 \mathrm{~min}$ a $40^{\circ} \mathrm{C}$, de $40^{\circ} \mathrm{C}$ a $90{ }^{\circ} \mathrm{C}$ a $3{ }^{\circ} \mathrm{C} \min ^{-1}, 90{ }^{\circ} \mathrm{C}$ a $300{ }^{\circ} \mathrm{C}$ a $12{ }^{\circ} \mathrm{C}$. $\min ^{-1}$ seguido por um período isotérmico de $5 \mathrm{~min}$. Injetor e detector foram mantidos a $240{ }^{\circ} \mathrm{C}$ e $300{ }^{\circ} \mathrm{C}$, respectivamente. Os padrões utilizados para coeluição foram adquiridos da Aldrich, Sigma, Fluka e Supelco e fazem parte da coleção de padrões da Sucocítrico Cutrale Ltda, Araraquara - SP. Os espectros de massas dos compostos identificados foram comparados com os da biblioteca "NIST" do equipamento.

\section{RMN de ${ }^{1} \mathbf{H}$}

RMN de ${ }^{1} \mathrm{H}, 400 \mathrm{MHz}$ : Espectrômetro Bruker ARX-400, solvente $\mathrm{CDCl}_{3}$ contendo TMS como referência interna.

\section{Transesterificação}

As reações dos triglicerídeos com $\mathrm{BF}_{3} /$ metanol para produzir os ésteres metílicos foram feitas a $80{ }^{\circ} \mathrm{C}$, durante 10 min e seguidas por extração com hexano após adição de água à mistura reacional.

\section{Leucoagaricus gongylophorus}

O fungo usado neste trabalho foi identificado como Leucoagaricus gongylophorus = Leucocoprinus gongylophorus (Heim), syn Rozites gongylophora (Möller). Ele foi isolado de um ninho de Atta sexdens e tem sido mantido no Centro de Estudo de Insetos Sociais da UNESP - Rio Claro.

\section{Ensaios de inibição do crescimento do fungo Leucoagaricus gongylophorus}

Os ensaios foram realizados de acordo com a metodologia desenvolvida por Pagnocca et al. ${ }^{14,15}$.

\section{Ensaios de ação inseticida tópica sobre Atta sexdens}

As operárias foram coletadas em lotes de 60 de um formigueiro mantido em laboratório, colocadas em bandejas plásticas, com as paredes laterais revestidas por "Teflon" e no interior foi mantido um pedaço de algodão embebido em água. Com o auxílio de uma pinça, cada formiga foi imobilizada para aplicação do extrato em seu pronoto. Para tanto, foi utilizada uma microseringa adaptada a um micrômetro, de modo a fornecer $1 \mu \mathrm{L}$ do extrato por formiga.

Dos óleos de sementes de laranja, limão e tangerina, foram testados os extratos obtidos com o solvente hexânico na concentração de $200 \mu \mathrm{g} / \mathrm{mL}$, diluídos em dois solventes, hexano e acetato de etila, para servir de veículo de transporte na aplicação.

Após as aplicações, as formigas foram colocadas em placas de Petri (100 mm X $15 \mathrm{~mm}$ ), forradas com papel de filtro, tendo sido distribuídas 6 formigas por placa e 10 placas por tratamento. As placas foram mantidas em estufa para B.O.D. à temperatura de $24^{\circ} \mathrm{C} \pm$ $1{ }^{\circ} \mathrm{C}$, com umidade relativa de no mínimo $70 \%$ e as formigas foram mantidas com dietas alimentares sólidas segundo Bueno et al. ${ }^{16}$.

A cada $24 \mathrm{~h}$, por um período de 25 dias, foram verificados os números de formigas mortas e realizadas as trocas das dietas alimentares.

Os dados foram analisados graficamente, através de curvas de sobrevivência e do teste estatístico "log rank test", utilizando o programa PRISMA 2.0 (Graph Pad Software) para a comparação das curvas de sobrevivência entre o grupo controle e os experimentais.

\section{RESULTADOS E DISCUSSÃO}

As extrações dos óleos das sementes de citros com hexano em aparelho "soxhlet" (Tabela 1) foram realizadas controlando os refluxos a cada 10 min. Foram extraídos 24,62; 24,27 e 28,35\% de óleos de limão siciliano, laranja e tangerina, respectivamente. Estes resultados encontram-se dentro da faixa obtida para Citrus sinensis, $C$. paradisi, $C$. aurantium, $C$. reticulata, $C$. aurantifolia e tangelo (um hibrido entre $C$. paradisi e $C$. reticulata), cujo conteúdo de óleo varia entre $24 \%$ e $41 \%{ }^{17}$. Estes resultados são, também, comparáveis àqueles obtidos por Stahl et al. ${ }^{4}$ usando a mesma metodologia, num 
Tabela 1. Extrações de óleos de limão siciliano, laranja e tangerina com hexano (270 mL) em aparelho "soxhlet".

\begin{tabular}{llllllllll}
\hline & \multicolumn{3}{c}{ Extração 1 } & \multicolumn{2}{c}{ Extração 2 } & \multicolumn{2}{c}{ Extração 3 } & \multicolumn{2}{c}{ Médias } \\
Material & $\begin{array}{l}\text { Sementes } \\
(\mathrm{g})\end{array}$ & $\begin{array}{l}\text { Extrato } \\
(\mathrm{g})\end{array}$ & $\begin{array}{l}\text { Sementes } \\
(\mathrm{g})\end{array}$ & $\begin{array}{l}\text { Extrato } \\
(\mathrm{g})\end{array}$ & $\begin{array}{l}\text { Sementes } \\
(\mathrm{g})\end{array}$ & $\begin{array}{l}\text { Extratos } \\
(\mathrm{g})\end{array}$ & $\begin{array}{l}\text { Sementes } \\
(\mathrm{g})\end{array}$ & $\begin{array}{l}\text { Extrato } \\
(\mathrm{g})\end{array}$ & $\begin{array}{c}\text { Rendimento } \\
\%\end{array}$ \\
\hline Limão siciliano & 23,00 & 5,63 & 23,85 & 5,90 & 22,24 & 5,49 & 23,03 & 5,67 & 24,62 \\
Laranja & 32,95 & 8,06 & 28,62 & 6,75 & 26,30 & 6,54 & 29,29 & 7,11 & 24,27 \\
Tangerina & 29,85 & 8,32 & 32,80 & 9,73 & 32,78 & 9,03 & 31,81 & 9,02 & 28,35 \\
\hline
\end{tabular}

Tabela 2. Extraçõs com fluido supercrítico de $\mathrm{CO}_{2}(8 \mathrm{~h})$ de sementes de limão siciliano (145 g) usando extrator "home made"

\begin{tabular}{lcc}
\hline Condições & Extrato $(\mathrm{g})$ & Rendimento $(\%)$ \\
\hline $15^{\circ} \mathrm{C}$ e $85 \cdot 10^{5} \mathrm{~Pa}$ & 0 & 0 \\
$40^{\circ} \mathrm{C}$ e $125 \cdot 10^{5} \mathrm{~Pa}$ & 2,39 & 1,65 \\
$60^{\circ} \mathrm{C}$ e $150 \cdot 10^{5} \mathrm{~Pa}$ & 1,10 & 0,76 \\
\hline TOTAL & 3,49 & 2,41 \\
\hline
\end{tabular}

estudo realizado para comparar a extração em aparelho "soxhlet" e por fluido supercrítico de $\mathrm{CO}_{2}$. Neste estudo eles obtiveram para sementes de soja, de girassol e de nabo os rendimentos de óleo 19,9, 38,4 e $40,1 \%$, respectivamente.

As condições de extração com fluido supercrítico de $\mathrm{CO}_{2}$ de sementes de citros foram determinadas através da adaptação daquelas descritas na literatura para outras sementes ${ }^{4,18-20}$. Essa técnica para as sementes de limão siciliano (Tabela 2), usando o equipamento extrator "home made", produziu pequena quantidade de óleo $(2,41 \%)$. A limitação de pressão de segurança deste extrator permitiu alcançar o máximo de $160.10^{5} \mathrm{~Pa}$ e mostrou que o rendimento foi mais baixo que o obtido por Stahl et al. ${ }^{4}$ para sementes de soja, de girassol e de nabo silvestre ("colza", nabo). Com a finalidade de melhorar o rendimento da extração, um extrator comercial foi usado e a produção de óleo aumentou. Contudo, as extrações foram feitas apenas com as sementes de laranja e tangerina e os rendimentos foram maiores, respectivamente $37,00 \%$ e $26,25 \%$ (Tabela 3 ). Na extensa literatura consultada, não há relato de estudos de extração de sementes de citros com fluido supercrítico e o estudo de Stahl et $a l .{ }^{4}$ para sementes de soja, girassol e nabo silvestre, que usaram condições de extrações similares e produziram $16,4,36,0$ e $39,3 \%$, respectivamente, que são ligeiramente menores que os obtidos por extração utilizando um aparelho "soxhlet".

Os extratos hexânicos de citros foram analisados por cromatografia gasosa, com injeção de $5 \mu \mathrm{L}$ do óleo e os compostos voláteis identificados por co-injeção de padrões e comparação de seus espectros de massa com os da biblioteca "NIST" do equipamento utilizado. Os resultados dessas análises mostram que os óleos de sementes extraídos com hexano são constituídos, além dos triglicerídeos, por vários compostos voláteis, tais como terpenóides e álcoois, ésteres e aldeídos graxos (Tabela 4). Os compostos voláteis das sementes de limão siciliano e de laranja são similares, com exceção das porcentagens relativas, enquanto que o óleo de tangerina é mais rico em número de compostos voláteis. Os compostos voláteis identificados são comuns na casca de citros $^{21}$. Entretanto, os óleos obtidos com extração por fluido supercrítico produziram apenas triglicerídeos.

Os triglicerídeos são os principais constituintes dos óleos de sementes vegetais. A composição em ácidos graxos dos triglicerídeos dos óleos das sementes de limão siciliano e laranja, obtidos por extração com hexano e por fluido supercrítico de $\mathrm{CO}_{2}$, foi determinada usando $\mathrm{CG} / \mathrm{EM}$, após reação dos triglicerídeos com $\mathrm{BF}_{3} / \mathrm{MeOH}$ dando origem aos ésteres metílicos. Os resultados dessas análises (Tabelas 5 e 6) mostram que os óleos obtidos por extração com fluido supercrítico possuem pequenas diferenças qualitativas quando comparados com aquele obtido pelo método tradicional de extração com solvente e estão dentro das faixas de porcentagens obtidas para diversas variedades de laranja, pomelo, tangerina, limão e lima ${ }^{22,23}$. Praticamente não existe diferença de porcentagem entre os constituintes dos óleos obtidos por extração com hexano e por fluido supercrítico de sementes de limão siciliano. As sementes de limão siciliano utilizadas nas duas metodologias de extração foram as mesmas. A ausência do ácido linoléico nos óleos de laranja obtidos por fluido supercrítico e a diferença em porcentagem entre os constituintes poderiam ser devidas ao material em cada análise não ser do mesmo lote. Este último resultado sugere a realização de novos estudos para determinar se esta variação está relacionada com a maturação do fruto (fator sazonal) ou se a região de coleta da semente é que determina esta variação.

Os ensaios de inibição do fungo simbionte com os extratos dos óleos das sementes obtidos por extração com solventes, hexano e diclorometano e por fluido supercrítico de $\mathrm{CO}_{2}$ (Tabela 7) mostram que o extrato hexânico das sementes de limão siciliano foi o mais ativo, resultando em $60 \%$ de inibição do crescimento do fungo Leucoagaricus gongylophorus na concentração de $200 \mu \mathrm{g} / \mathrm{mL}$. Essa atividade foi bastante reduzida quando o ensaio foi realizado com metade da concentração. A maior atividade antifúngica observada com o extrato hexânico das sementes de limão siciliano pode estar relacionada com a presença dos constituintes do seu óleo volátil (Tabela 4) e estudos com os mesmos devem ser realizados para confirmar essa hipótese. Estudos realizados com ácidos graxos livres, oriun-

Tabela 3. Extrações utilizando fluido supercrítico de $\mathrm{CO}_{2}$ de sementes de laranja e tangerina $(4 \mathrm{~g})$ usando extrator comercial a $40{ }^{\circ} \mathrm{C}$

\begin{tabular}{lcccccc}
\hline Semente & Pressão e tempo & Extração $1(\mathrm{~g})$ & Extração $2(\mathrm{~g})$ & Extração $3(\mathrm{~g})$ & Médias $(\mathrm{g})$ & $\%$ médias \\
\hline LARANJA & $280 \cdot 10^{5} \mathrm{~Pa}, 60 \mathrm{~min}$ & 0,05 & 0,06 & 0,07 & 0,06 & 1,50 \\
& $420 \cdot 10^{5} \mathrm{~Pa}, 12 \mathrm{~min}$ & 0,54 & 0,72 & 0,75 & 0,67 & 16,75 \\
& $490 \cdot 10^{5} \mathrm{~Pa}, 8 \mathrm{~min}$ & 0,73 & 0,77 & 0,76 & 0,75 & 18,75 \\
& TOTAL & 1,32 & 1,55 & 1,58 & 1,48 & 37,00 \\
\hline TANGERINA & $280 \cdot 10^{5} \mathrm{~Pa}, 60 \mathrm{~min}$ & 0,07 & $\mathrm{NE}$ & $\mathrm{NE}$ & 0,02 & 0,50 \\
& $420 \cdot 10^{5} \mathrm{~Pa}, 12 \mathrm{~min}$ & 0,49 & 0,56 & 0,54 & 0,53 & 13,25 \\
& $490 \cdot 10^{5} \mathrm{~Pa}, 8 \mathrm{~min}$ & 0,40 & 0,48 & 0,61 & 0,50 & 12,50 \\
& TOTAL & 0,96 & 1,04 & 1,15 & 1,05 & 26,25 \\
\hline
\end{tabular}


Tabela 4. Compostos voláteis identificados por co-injeção nos extratos das sementes de limão siciliano, laranja e tangerina

\begin{tabular}{|c|c|c|c|}
\hline Compostos & $\begin{array}{c}\text { Limão siciliano } \\
(\% *)\end{array}$ & $\begin{array}{l}\text { Laranja } \\
(\%)\end{array}$ & $\begin{array}{c}\text { Tangerina } \\
(\%)\end{array}$ \\
\hline Heptan-1-ol & 40,23 & 9,87 & 0,29 \\
\hline 2-Furaldeido & A & A & 0,05 \\
\hline 2-Metilpropan-1-ol & 1,87 & $\mathrm{~T}$ & $\mathrm{~T}$ \\
\hline Hexan-3-ol & A & A & 0,06 \\
\hline Acetal** & $\mathrm{A}$ & A & 0,04 \\
\hline Acetato de etila & 0,18 & $\mathrm{~T}$ & $\mathrm{~T}$ \\
\hline Acetato de geranila & 0,08 & A & 0,02 \\
\hline Acetato de linalila & A & A & 0,02 \\
\hline Acetato de nerila & 0,06 & A & 0,07 \\
\hline$\alpha$-Pineno & 0,22 & A & 0,06 \\
\hline$\alpha$-Terpineol & A & A & 0,13 \\
\hline$\beta$-Citronelol & A & A & 0,01 \\
\hline$\beta$-Mirceno & 2,73 & $\mathrm{~T}$ & 0,54 \\
\hline$\beta$-Pineno & A & A & 0,45 \\
\hline Canfeno & 1,37 & 1,58 & 0,06 \\
\hline Cumeno & 0,19 & $\mathrm{~T}$ & $\mathrm{~T}$ \\
\hline D-Limoneno & 4,16 & 3,44 & 3,77 \\
\hline Decan-1-ol & A & A & 0,05 \\
\hline Dodecan-1-ol & A & A & 0,03 \\
\hline$\gamma$-Terpineno & $\mathrm{A}$ & A & 0,44 \\
\hline Geranial & 0,13 & A & 0,06 \\
\hline Heptanal & A & A & 0,02 \\
\hline Hexanal & 5,22 & $\mathrm{~T}$ & 0,06 \\
\hline Linalol & A & A & 0,02 \\
\hline Neral & A & A & 0,04 \\
\hline Nerol & A & A & A \\
\hline Nonanal & A & $\mathrm{A}$ & 0,04 \\
\hline Octanal & 4,02 & 1,28 & $\mathrm{~T}$ \\
\hline p-Cimeno & A & A & 0,03 \\
\hline Terpinoleno & A & A & 0,02 \\
\hline Trans-Hex-2-enal & 1,06 & $\mathrm{~T}$ & 0,03 \\
\hline Valenceno & A & A & 0,06 \\
\hline
\end{tabular}

$\mathrm{A}=$ ausente, $\mathrm{T}=$ Traço, $*$ Porcentagem relativa à soma das áreas dos picos no CG, $* *$ Acetal $=$ dietilacetaldeido acetal $($ Merck Index $)$

Tabela 5. Composição de ácidos graxos, identificados como ésteres metílicos, dos triglicerídeos dos óleos das sementes de limão siciliano, laranja e tangerina extraídos com hexano

\begin{tabular}{lccc}
\hline $\begin{array}{l}\text { Ácidos } \\
\text { graxos }\end{array}$ & $\begin{array}{c}\text { Limão siciliano } \\
(\% *)\end{array}$ & $\begin{array}{c}\text { Laranja } \\
(\%)\end{array}$ & $\begin{array}{c}\text { Tangerina } \\
(\%)\end{array}$ \\
\hline Ác. palmítico & 29,8 & 53,9 & 38,6 \\
Ac. linoléico & 24,1 & 29,6 & 30,5 \\
Ac. linolênico & 38,7 & 13,5 & 19,9 \\
Ac. oléico & 2,4 & 0,7 & 2,1 \\
Ac. esteárico & 5,0 & 2,3 & 8,9 \\
\hline
\end{tabular}

* Porcentagem relativa à soma das áreas dos picos no CG dos do extrato hexânico das folhas de Canavalia ensiformis, mostraram que a fração que contém os ácidos saturados com $\mathrm{C}_{11}, \mathrm{C}_{17}, \mathrm{C}_{19}$, $\mathrm{C}_{22}$ e $\mathrm{C}_{23}$ entre os mais abundantes na mistura, apresenta atividade antifúngica superior àquela que contém ácidos semelhantes aos encontrados nos triglicerídeos dos citros analisados neste trabalho ${ }^{12}$.

Inicialmente, para os ensaios in vitro com as saúvas, utilizando os óleos das sementes dos citros extraídos com hexano, foram realizadas as comparações entre as curvas de sobrevivência das formigas que receberam os solventes e aquelas que receberam somente a dieta alimentar sem nenhum tratamento, para verificar o possível efeito do solvente. As análises mostraram que não houve diferenças significativas entre os grupos, indicando que os solventes hexano e acetato de etila, usados na diluição e no transporte do óleo, não causaram efeitos tóxicos às formigas.

Os ensaios para verificar a ação inseticida com óleos extraídos com hexano de sementes de laranja, limão e tangerina e diluídos com este mesmo solvente para aplicação mostraram que não houve diferença significativa nas sobrevivências das formigas. Os valores medianos foram: controle: 17 dias, laranja: 19 dias, limão: 21 dias e tangerina: 14 dias. Por outro lado, quando os óleos foram diluídos com acetato de etila para aplicação, os valores diferiram significativamente: controle: 20 dias, laranja 13 dias $\left(x^{2}=14,84, p<0,01\right)$ (resultado do "log rank test" entre as curvas de sobrevivência do controle $X$ extrato); limão 15 dias $\left(x^{2}=4,29, p=0,0384\right)$ e tangerina de 10 dias $\left(x^{2}=9,637, p<0,01\right)$, demonstrando atividade tóxica.

\section{CONCLUSÕES}

Os resultados deste trabalho indicam que as extrações usando fluido supercrítico de $\mathrm{CO}_{2}$ apresentaram maior seletividade, quando comparadas com as extrações com hexano em aparelho "soxhlet", produzindo maior quantidade de óleo e estes foram claros e não apre-

Tabela 6. Composição de ácidos graxos, identificados como ésteres metílicos, dos triglicerídeos dos óleos das sementes de limão siciliano e laranja extraídos com fluido supercrítico de $\mathrm{CO}_{2}$

\begin{tabular}{lcc}
\hline Ácidos graxos & Limão siciliano $(\% *)$ & Laranja $(\%)$ \\
\hline Ác. palmítico & 29,3 & 58,7 \\
Ac. linoléico & 23,9 & $\mathrm{~A}$ \\
Ac. linolênico & 38,1 & 24,8 \\
Ac. oléico & 1,9 & 2,0 \\
Ac. esteárico & 5,8 & 12,9 \\
Desconhecido1** & 0,5 & 0,8 \\
Desconhecido 2** & 0,5 & 0,8 \\
\hline
\end{tabular}

$\overline{\mathrm{A}=\text { Ausente, }} *$ Porcentagem relativa à soma das áreas dos picos no CG, **Não identificados por comparação dos espectros de massas e presentes na faixa de tempo de retenção dos ésteres metílicos de ácidos graxos

Tabela 7. Porcentagem de inibição do crescimento do fungo Leucoagaricus gongylophorus, simbionte de formigas cortadeiras, em diferentes concentrações $(\mu \mathrm{g} / \mathrm{mL})$ dos óleos das sementes de citros extraídos com hexano, diclorometano e com fluido supercrítico de $\mathrm{CO}_{2}$

\begin{tabular}{|c|c|c|c|c|c|c|}
\hline \multirow[t]{2}{*}{ Variedade } & \multicolumn{2}{|c|}{ Extrato hexânico } & \multicolumn{2}{|c|}{ Extrato diclorometânico } & \multicolumn{2}{|c|}{$\begin{array}{l}\text { Extrato obtido por fluido } \\
\text { supercrítico de } \mathrm{CO}_{2}\end{array}$} \\
\hline & $\mu \mathrm{g} / \mathrm{mL}$ & Inibição (\%) & $\mu \mathrm{g} / \mathrm{mL}$ & Inibição (\%) & $\mu \mathrm{g} / \mathrm{mL}$ & Inibição (\%) \\
\hline Tangerina & 200 & 20 & 200 e 100 & 20 & NT & NT \\
\hline Laranja & 200 & 20 & 200 e 100 & 20 & 1000 & 40 \\
\hline Limão siciliano & $\begin{array}{l}200 \\
100\end{array}$ & $\begin{array}{l}60 \\
10\end{array}$ & 200 e 100 & 20 & 200 & 20 \\
\hline
\end{tabular}

NT = não testado 
sentaram ácidos graxos livres. A presença de ácidos graxos livres é usual em óleo vegetal e torna o mesmo de baixa qualidade ${ }^{23}$. Portanto, o uso de fluido supercrítico de $\mathrm{CO}_{2}$ para obter óleos contendo apenas triglicerídeos é uma técnica recomendável.

As atividades dos óleos das sementes de citros sobre o crescimento do fungo simbionte das formigas cortadeiras foram de baixa toxicidade, quando comparados com os de extratos de plantas estudadas anteriormente por nós ${ }^{11,12}$, assim como de sesamina, que apresentou atividade inibitória na concentração de $70 \mu \mathrm{g} / \mathrm{mL}^{15}$.

Os óleos das sementes de laranja, limão e tangerina apresentaram efeitos tóxicos para as saúvas nas condições testadas e foram extremamente dependentes do solvente utilizado como veículo de aplicação. Dentre os três óleos de sementes testados, o mais promissor para possível aplicação como inseticida para formigas cortadeiras foi o da tangerina.

Estudos anteriores de ação inseticida realizados com triglicerídeos e com ácidos graxos livres indicaram que os ácidos graxos mostraram maior atividade que os triglicerídeos e esta foi aumentada pela presença de triglicerídeos, os quais devem aumentar a absorção do ácido ${ }^{13}$. Portanto, devido às grandes quantidades destes óleos que podem ser obtidas anualmente e ao uso de casca de citros como um dos constituintes atrativos das iscas para saúvas, novos estudos devem ser realizados para verificar a possibilidade do uso destes óleos no controle de formigas cortadeiras, mesmo em altas concentrações ou associados a ácidos graxos livres.

\section{AGRADECIMENTOS}

Os autores agradecem ao Conselho Nacional de Desenvolvimento Científico e Tecnológico (CNPq), Coordenação de Pessoal de Nível Superior (CAPES), PRONEX/MCT-FINEP e Fundação de Amparo à Pesquisa do Estado de São Paulo (FAPESP), pelo suporte financeiro e bolsas (CNPq), assim como a Sucocítrico Cutrale Ltda por ceder os equipamentos analíticos, extrator "home made" e padrões.

\section{REFERÊNCIAS}

1. Malavolta, E.; Netto Violante, A.; Nutrição Mineral, Calagem, Gessagem e Adubação dos Citros, Centro de Energia Nuclear na Agricultura Universidade de São Paulo, Ed. Gráfica Nagy Ltda: Piracicaba, 1989.

2. Marques, D.S.; Dissertação de Mestrado, Universidade Estadual de Campinas, Brasil, 1997.

3. United Nations. FAO; Nutritional Studies. Amino Acid Content of Foods and Biological Data on Proteins, 24, Rome, 1970.

4. Stahl, E.; Schutz, E.; Mangold, H. K.; J. Agric. Food Chem. 1980, 28, 1153.

5. Wilson. E. O.; Behavioral Ecol. and Sociobiology 1980, 7, 143.

6. Martin, M. M.; Science 1970, 169, 16.

7. Fisher, P. J.; Stradling, D. J.; Pegler, D. N.; Mycologist 1994, 8, 128.

8. Fisher, P. J.; Stradling, D. J.; Pegler, D. N.; Mycol. Res. 1994, 98, 884.

9. Quinlan, R. J.; Cherrett, J. M.; Ecol. Entomol. 1979, 4, 151.

10. Bass, M.; Cherrett, J. M.; Physiol. Ent. 1995, 20, 1.

11. Bueno, O. C.; Hebling-Beraldo, M. J. A.; Silva, O. A. da; Pagnocca, F. C.; Fernandes, J. B.; Vieira, P. C. Em Applied Myrmecology, A World Perspective; Meer, R. K. V.; Jaffe, K.; Cedeno, A., eds.; Westview Press, 1990, p. 426.

12. Monteiro, M. R.; Torkomian, V. L. V.; Pagnocca, F. C.; Vieira, P. C.; Fernandes, J. B.; da Silva, M. F. G. F.; Bueno, O. C.; Hebling, M. J. A.; An. Acad. Bras. Ci. 1998, 70, 733.

13. Morini, M. S. C.; Bueno, O. C.; Hebling, M. J. A.; Pagnocca, F. C.; Bacci Jr., M.; Fernandes, J. B.; Vieira, P. C.; Naturalia 1999, 24, 327.

14. Pagnocca, F. C.; Silva, O. A.; Hebling-Beraldo, M. J. A.; Bueno, O. C.; Fernandes, J. B.; Vieira, P. C.; Bull. Entomol. Res. 1990, 80, 349.

15. Pagnocca, F. C.; Ribeiro, S. B.; Torkomian, V. L. V.; Hebling-Beraldo, M. J. A.; Bueno, O. C.; Silva, O. A.; Fernandes, J. B.; Vieira, P. C.; Da Silva, M. F. G. F.; Ferreira, A. G.; J. Chem. Ecol. 1996, 22, 1325.

16. Bueno, O. C.; Morini, M. S. C.; Pagnocca, F. C.; Hebling, M. J. A.; Silva, O. A.; An. Soc. Entomol. Brasil 1997, 26, 107.

17. Ajewole, K.; Adeyeye, A.; Food Chem. 1993, 47, 77.

18. Bevan, C. D.; Marshall, P. S.; Nat. Prod. Rep. 1994, 11, 451.

19. Bruner, G.; Peter, S.; Sep. Sci. Technol. 1982, 17, 199.

20. Eggers, R.; Sievers, U.; Stein, W.; J. Am. Org. Chem. Soc. 1985, 62, 1222.

21. Kefford, J. E.; Chandler, B. V.; The Chemical Constituents of Citrus Fruits, Adv. in Foods Res., Supplements, Academic Press: New York, 1970.

22. Hendrickson, R.; Kesterson, J. W.; J. Am. Oil Chem. Soc. 1963, 40, 746.

23. Braddock, R. J.; Kesterson, J. W.; Agr. Esp. Sta. Bull. 1973, 756, 30. 